\title{
Intraluminal Behavior of Various Transporter Substrates in the Rat Gastrointestinal Tract
}

Yusuke Tanaka, Taiki Harada, Kazuhiro Ito, Takanori Kurakazu, Satoshi Kasaoka

Laboratory of Pharmaceutics, Faculty of Pharmaceutical Sciences, Hiroshima International University, Hiroshima, Japan

Corresponding author: Yusuke Tanaka, Laboratory of Pharmaceutics, Faculty of Pharmaceutical Sciences, Hiroshima International University, 5-1-1 Hiro-koshingai, Kure, Hiroshima, 737-0112, Japan; email: y-tanaka@ phirokoku-u.ac.jp

Received, September 3, 2021; Revised, November 15, 2021; Accepted, November 18, 2021; Published. November 21, 2021

\begin{abstract}
Purpose: The aim of this study was to evaluate the intraluminal behavior of various transporter substrates in different regions of the gastrointestinal (GI) tract. Methods: Drug solutions containing nonabsorbable FITC-dextran 4000 (FD-4), were orally administered to rats. Residual water was sampled from the GI regions to measure the luminal drug concentration. Results: Cephalexin (CEX), a substrate of the proton-coupled oligopeptide transporter, was absorbed rapidly, and no drug was detected in the lower small intestine. Saquinavir (SQV) was primarily absorbed in the upper region. However, unlike CEX, SQV was detected even in the lower segment probably due to the efflux of SQV via P-glycoprotein (P-gp). The concentration of methotrexate (MTX) showed a similar pattern to that of non-absorbable FD-4. The low absorption of MTX was probably due to efflux via several efflux transporters, and the limited expression of proton-coupled folate transporter, an absorptive transporter for MTX, in the upper region. Conclusion: This study revealed that the luminal concentration pattern of each drug differed considerably depending on the site because of the different absorption properties and luminal volumes. Although further investigation using a specific transporter inhibitor or transporter-knockout animals are necessary to clarify the actual contribution of each transporter to the drug absorption, this information will be valuable in evaluating transporter-mediated drug absorption in in vitro transport studies for ensuring optimal drug concentrations.
\end{abstract}

\section{INTRODUCTION}

A variety of influx and efflux transporters are expressed in the small intestine (1-3). Intestinal permeability of transporter substrates changes depending on intraluminal drug concentration $(4,5)$, sometimes leading non-linear bioavailability $(6,7)$. Total intestinal fluid volume was often used to estimate average luminal drug concentration for evaluation of transporter-mediated drug uptake $(8,9)$. In our previous report, however, the luminal volume was drastically different depending on the segments of the gastrointestinal (GI) tract due to fluid absorption and secretion $(10,11)$, indicating that the use of total intestinal volume to estimate average luminal drug concentration may have a risk of failure in the evaluation of drug absorption, especially via transporters with site-specific expression [e.g. Proton-coupled folate transporter (PCFT) and Multidrug resistance-associated protein 2 (MRP2) that are primarily expressed in proximal small intestine $(2,12)$ and P-glycoprotein (P-gp) that is primarily expressed in distal small intestine (2)]. Thus, understanding the behavior of transporter substrates in various segments of the GI tract is very important to evaluate and predict carrier-mediated absorption more accurately. Intraluminal drug behaviors in humans have been reported by several groups (13-15). However, since an intubation method was typically used for luminal fluid sampling, it is technically difficult to obtain a sample from the lower segment. Therefore, relevant information, especially for luminal behavior in the lower region (e.g., lower jejunum and ileum) is still limited. In our previous study, intraluminal concentrations of several drugs that are passively absorbed were measured by direct sampling of residual fluid from individual GI segments including lower regions $(11,16)$, and it is elucidated that intraluminal behavior differed depending on physicochemical properties of drugs (e.g., solubility and permeability). It is considered that our methodology is useful to elucidate concentration patterns of transporter substrates in different GI segments.

In this study, to quantitatively measure the luminal concentrations as a function of time in individual GI segments, various transporter substrates were orally administered to rats. In 
addition, non-absorbable FITC-dextran (FD-4) was co-administered as a control, and the drug absorption behavior was accurately determined by comparing the luminal concentrations of FD-4 and other drugs. Cephalexin (CEX), a substrate of the peptidetransporter 1 (PEPT1) (17); saquinavir (SQV), a substrate of P-gp (18) and MRP2 (2); and methotrexate (MTX), a substrate of PCFT (12), reduced folate carrier 1 (RFC1) (19), breast cancer resistance protein (BCRP) (20), MRP2 (12), and Pgp $(21,22)$ were selected as model drugs to evaluate the impact of absorption behavior via various transporters on luminal concentration pattern of drugs.

ABBREVIATION. BA: bioavailability; BCRP: breast cancer resistance protein; CEX: Cephalexin ; FD-4 : FITC-dextran ; GI : gastrointestinal ; MTX: Methotrexate; MRP2: Multidrug resistance-associated protein 2; P-gp: P-glycoprotein; PCFT: proton-coupled folate transporter; PEPT1: peptide-transporter 1; SQV: Saquinavir

\section{MATERIALS AND METHODS}

\section{Materials}

FD-4 was purchased from Sigma-Aldrich (St. Louis, MO, USA). CEX, ritonavir, and metoprolol tartrate were purchased from Tokyo Chemical Industry Co., Ltd. (Tokyo, Japan). Saquinavir mesylate, MTX, and antipyrine were purchased from FUJIFILM Wako Pure Chemical Corporation (Osaka, Japan). All other reagents were analytical-grade commercial products.

\section{Luminal concentrations of FD-4 and drugs, and the plasma concentrations of drugs after oral administration}

All experimental procedures were approved by the Animal Experimentation Ethics Committee of the Hiroshima International University and were in accordance with the Guide to the Care and Use of Experimental Animal Care. Male Sprague Dawley rats (244-298 g) were sourced from Japan SLC (Hamamatsu, Japan). Rats were fasted overnight prior to the experiments; water was provided ad libitum but limited to $2 \mathrm{~h}$ prior to administration.

The luminal concentration of FD-4 and drugs was measured as described previously $(10,11)$. CEX and MTX were dissolved in Milli-Q water at concentrations of 1.0 and $0.025 \mathrm{mg} / \mathrm{mL}$, respectively. Saquinavir mesylate was dissolved to a concentration of $0.2 \mathrm{mg} / \mathrm{mL}$ as free form in $\mathrm{HCl}$ solution ( $\mathrm{pH} 2.4$ ) instead of Milli-Q water due to solubility problems. Subsequently, FD-4 was solubilized in the respective drug solutions to a final concentration of $200 \mu \mathrm{M}$.

Each solution $(1 \mathrm{~mL})$ was orally administered to rats. The doses were $0.2 \mu \mathrm{mol}$ for FD-4 and 1.0, 0.2 and $0.025 \mathrm{mg}$ for CEX, SQV and MTX, respectively. After 5, 10, 20, 30, 60, 90, 120, and 150 min, blood samples were collected from the right jugular vein, and rats were euthanized by injecting a potassium chloride solution $(22.5 \mathrm{mg} / \mathrm{rat})$ into the left jugular vein under anesthesia with isoflurane. Subsequently, each GI segment was dissected immediately to collect residual water from the stomach, duodenum (approximately 2-cm from the stomach), upper jejunum (approximately 20 -cm from the stomach), lower jejunum (approximately $60-\mathrm{cm}$ from the stomach), and ileum (approximately $10-\mathrm{cm}$ from the cecum). Three and four rats were used in each time point for CEX and for SQV and MTX, respectively. The samples were diluted with Milli-Q water for CEX and MTX, and with dimethyl sulfoxide for SQV. The samples were further diluted using appropriate solvents: $50 \mathrm{mM}$ Tris buffer $(\mathrm{pH}$ 8.0) for FD-4, Milli-Q water for CEX and MTX, and $50 \%$ methanol for SQV determination. Furthermore, CEX samples were mixed with an internal standard $(1.0 \mu \mathrm{g} / \mathrm{mL}$ antipyrine in $50 \%$ methanol) for LCMS/MS analysis. SQV and MTX in the samples were analyzed by HPLC.

\section{Collecting and Processing blood samples}

CEX and MTX were solubilized in saline at 1.0 and $0.025 \mathrm{mg} / \mathrm{mL}$, respectively, and saquinavir mesylate was dissolved at $0.2 \mathrm{mg} / \mathrm{mL}$ as free $\mathrm{SQV}$ in saline containing polyethylene glycol 400 at $30 \% \mathrm{v} / \mathrm{v}$. Subsequently, each solution $(0.5 \mathrm{~mL})$ was administered into the jugular vein of rats. Thereafter, blood samples were taken from the cannulated femoral artery at $5,10,20,30,40,50,60,75$, and 90 $\min$.

Blood samples collected after oral and intravenous administration of drugs were centrifuged and the obtained plasma $(50 \mu \mathrm{L})$ was mixed with $50 \mu \mathrm{L}$ of methanol and $50 \mu \mathrm{L}$ of an internal standard (1.0 $\mu \mathrm{g} / \mathrm{mL}$ antipyrine, $1.0 \mu \mathrm{g} / \mathrm{mL}$ metoprolol and 0.1 $\mu \mathrm{g} / \mathrm{mL}$ ritonavir in methanol for CEX, MTX and SQV, respectively.). Precipitated proteins were removed by centrifugation, and the supernatant was quantified by LC-MS/MS.

\section{Oral bioavailability (BA)}

As described in a previous report (11), oral BA-time profiles of various drugs were calculated using decon.xls, a Microsoft excel incorporating a program for deconvolution. This program is currently 
available

at

http://www4.tokai.or.jp/DMPK/program.html. The plasma concentration-time courses after oral administration of the three drugs were used as an output function, and those after the intravenous administration were used as an input function (23).

\section{Quantification of FD-4 and drugs}

FD-4 in the luminal samples was quantified using a multilabel luminescence counter (ARVO-MX, PerkinElmer Japan, Kanagawa, Japan), at wavelengths of 492 and $535 \mathrm{~nm}$ for excitation and emission, respectively.

MTX and SQV in luminal samples were determined using a Shimadzu HPLC system with a UV detector (SPD-20A; Shimadzu Corporation, Kyoto, Japan). An analytical column (YMC-Pack Pro C18, 150×6.0 mm I.D.,_YMC Co., Ltd. Japan) was used at $40^{\circ} \mathrm{C}$; the mobile phase consisted of a 50 $\mathrm{mM}$ phosphate buffer $(\mathrm{pH} 2.5)$ and acetonitrile $(85: 15 \mathrm{v} / \mathrm{v})$ or $50 \mathrm{mM}$ phosphate buffer $(\mathrm{pH} 6.0)$ and acetonitrile $(35: 65 \mathrm{v} / \mathrm{v})$ were run isocratically at a flow rate of $1 \mathrm{~mL} / \mathrm{min}$ for MTX and SQV, respectively. MTX and SQV were detected at wavelengths of 304 and $240 \mathrm{~nm}$, respectively.

Drugs in plasma samples and CEX in luminal samples were measured using an LC-MS/MS (LCMS-8040, Shimadzu, Kyoto, Japan). A Zorbax Eclipse XDB-C18 column $(2.1 \times 50 \mathrm{~mm}$, I.D., $5 \mu \mathrm{m})$ was used at $40^{\circ} \mathrm{C}$. The mobile phases consisted of $0.1 \%$ formic acid in Milli-Q water and methanol at $4: 1$ and $17: 3 \mathrm{v} / \mathrm{v}$ were used for CEX and antipyrine (IS) and for MTX and metoprolol (IS) quantifications, respectively. For SQV and ritonavir (IS) determination, $10 \mathrm{mM}$ ammonium formate containing $0.1 \%$ formic acid in Milli-Q water and methanol $(7: 13 \mathrm{v} / \mathrm{v})$ was used. The transition monitored for quantification was $\mathrm{m} / \mathrm{z} 348.0>157.9$ for CEX, $189.0>56.1$ for antipyrine, $455.1>308.1$ for MTX, $268.2>116.1$ for metoprolol, $671.3>570.2$ for SQV, and 721.1>296.2 for ritonavir.

\section{RESULTS}

\section{Luminal concentration-time profiles of FD-4 and experimental drugs}

The luminal concentrations of FD-4 and experimental drugs are shown in Figures 1-3. The vertical axis displays concentration as the percentage in the initial dosing concentration of FD-4 $(200 \mu \mathrm{M})$ and drugs $(1.0,0.2$, and $0.025 \mathrm{mg} / \mathrm{mL}$ for CEX, SQV, and MTX, respectively).

The gastric concentrations of FD-4 and CEX at $5 \mathrm{~min}$ were $54.3 \pm 15.6 \%$ and $51.5 \pm 17.8 \%$, respectively (Figure 1). Thereafter, the gastric CEX concentration decreased parallelly with the FD-4 concentration over time. In the duodenum and upper jejunum, the concentrations of CEX at 5 and $10 \mathrm{~min}$ were lower than those of non-absorbable FD-4, indicating that CEX was absorbed to some extent in these segments. In the lower jejunum and ileum, no CEX was detected, indicating that CEX was completely absorbed before it reached these regions. For FD-4, the maximum concentrations in the upper jejunum, lower jejunum and ileum (136.3 \pm 93.9, $245.8 \pm 284.6$ and $359.5 \pm 255.0 \%$, respectively) were above the dosing concentration. This was attributed to the condensation of FD-4 by water absorption transitioned to the lower segment, which is consistent with our previous study (10).

The luminal SQV concentration-time courses are shown in Figure 2. SQV is known as a substrate of P-gp and MRP2, efflux transporters (2). After oral administration, SQV rapidly passed through the stomach and duodenum. In the upper jejunum, SQV was absorbed to some extent until it reached this region, leading lower concentration of SQV than that of FD-4. In the lower jejunum and ileum, some SQV was detected (the $\mathrm{C}_{\max }$ was $61.8 \pm 33.9$ and $86.1 \pm$ $55.2 \%$ in the lower jejunum and ileum, respectively), unlike in the case of CEX. In our previous study, a simulated intestinal fluid was produced based on concentrations of bile acids $(51 \mathrm{mM})$ and phospholipids $(3.7 \mathrm{mM})$ in the jejunum of fasted rats (24), which values are much higher than those in the human small intestine (25). The solubility of SQV in the rat simulated intestinal fluid was $264.2 \pm 8.6$ $\mu \mathrm{g} / \mathrm{mL}$ (26).Although SQV is a lipophilic base, the concentrations of SQV in each segment of the small intestine were below the solubility values, and thus it is expected that SQV was present in a solubilized state in the small intestine.

The concentrations of FD-4 and MTX in the stomach at $5 \mathrm{~min}$ were $54.5 \pm 21.0 \%$ and $50.2 \pm$ $24.9 \%$, respectively, and decreased in the same manner (Figure 3). In the small intestine, the $C_{\max }$ values of MTX were $30.6 \pm 35.5,165.4 \pm 46.3,246.7$ \pm 155.3 and $232.3 \pm 152.1 \%$, in the duodenum, upper jejunum, lower jejunum and ileum, respectively. These concentration values of MTX were only slightly lower than those of FD-4, indicating low absorption of MTX from the GI tract. 

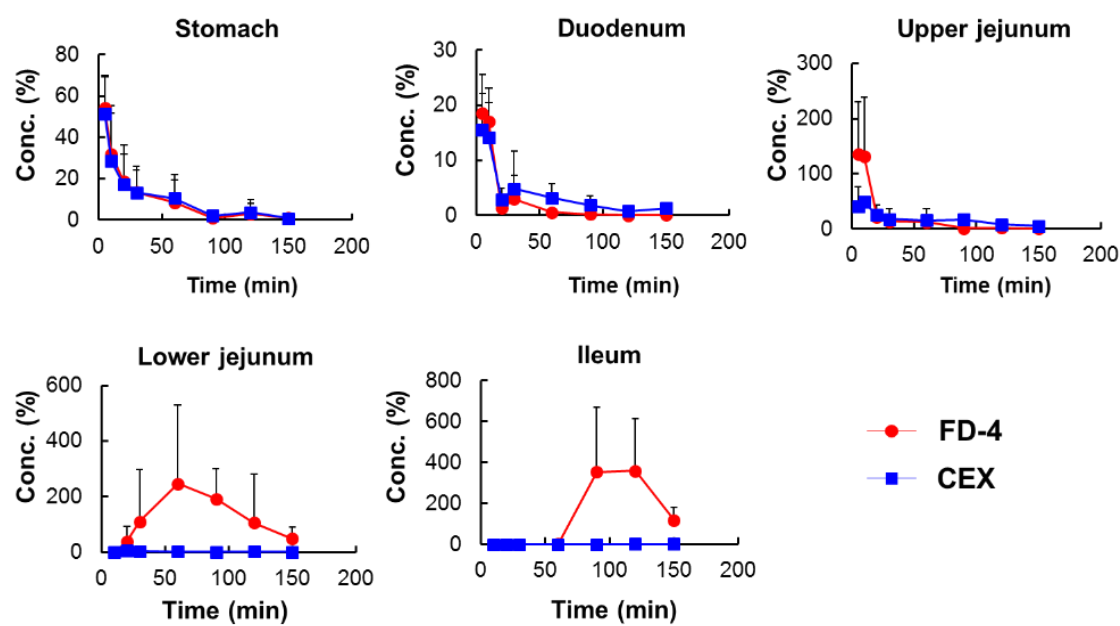

- CEX

Figure 1. Luminal concentration-time profiles in different intestinal segments of FD-4 and CEX after oral administration. Results are expressed as mean values, with vertical bars showing S.D. of three experiments. The vertical axes are expressed as the percentage of the respective dosing concentrations (i.e., FD-4: $200 \mu \mathrm{M}$ or CEX: 1.0 mg/mL).
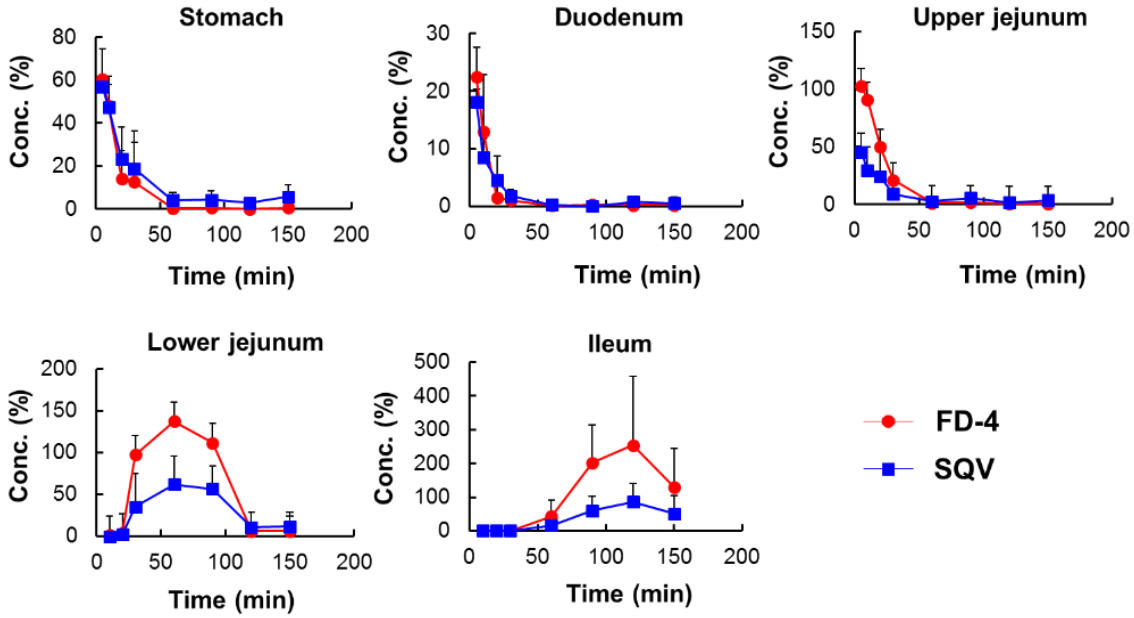

- SQV

Figure 2. Luminal concentration-time profiles in different intestinal segments of FD-4 and SQV after oral administration. Results are expressed as mean values, with vertical bars showing S.D. of four experiments. The vertical axes are expressed as the percentage of the respective dosing concentrations (i.e., FD-4: $200 \mu \mathrm{M}$ or SQV: $0.2 \mathrm{mg} / \mathrm{mL}$ ).
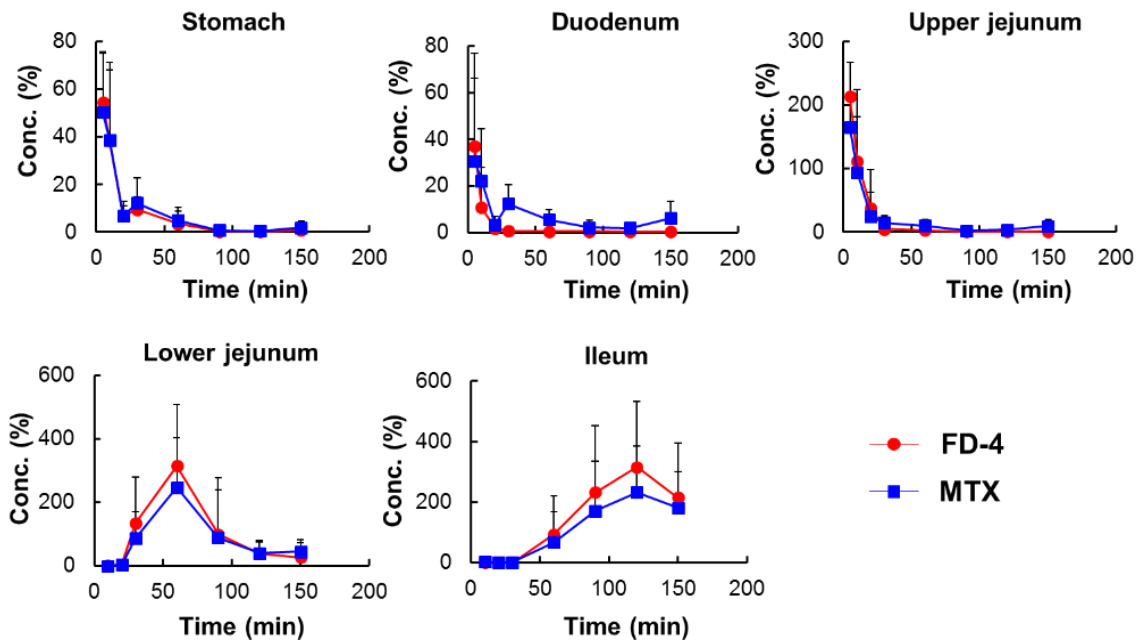

Figure 3. Luminal concentration-time profiles in different intestinal segments of FD-4 and MTX after oral administration. Results are expressed as mean values, with vertical bars showing S.D. of four experiments. The vertical axes are expressed as the percentage of the respective dosing concentrations (i.e., FD-4: $200 \mu \mathrm{M}$ or MTX: 0.025 mg/mL). 


\section{Time profiles of plasma concentrations and oral BA of experimental drugs}

The plasma concentration-time profiles after oral and intravenous administrations of drugs were shown in Figure 4. The concentrations at $5 \mathrm{~min}$ after intravenous administrations were $4.44 \pm 0.25 \mu \mathrm{g} / \mathrm{mL}$, $88.6 \pm 6.51 \mathrm{ng} / \mathrm{mL}$ and $80.7 \pm 8.47 \mathrm{ng} / \mathrm{mL}$ for CEX, SQV and MTX, respectively, and decreased rapidly with time. In the oral administration groups, the plasma concentration reached the peak at 30,5 and 10 min for CEX, SQV and MTX, respectively, and the $\mathrm{C}_{\max }$ values were $2.39 \pm 0.57 \mu \mathrm{g} / \mathrm{mL}$ for CEX, $8.13 \pm 4.12 \mathrm{ng} / \mathrm{mL}$ for SQV and $32.9 \pm 49.9 \mathrm{ng} / \mathrm{mL}$ for MTX.

The time profiles of oral BA were calculated by the deconvolution method using the plasma concentration-time courses of drugs after oral and intravenous administrations (Figure 5). CEX was continuously absorbed, and the BA reached $85.2 \%$ at 150 min after oral administration. In the case of SQV, the BA rapidly increased to $4.7 \%$ at $20 \mathrm{~min}$, and thereafter, the absorption rate decelerated, suggesting that SQV was primarily absorbed from the upper region. Similarly, the absorption rate of MTX was faster until $20 \mathrm{~min}$ and decreased in the later time period.

\section{DISCUSSION}

To accurately evaluate and predict carrier-mediated absorption, it is essential to understand the actual concentration pattern of transporter substrates in the GI tract and applying this information in the PBPK model and/or in vitro transport experiments. The present study therefore attempted to evaluate absorption behavior of various transporter substrates in the GI tract and its impact on the luminal drug concentration profiles.

After gastric emptying of CEX following oral administration, although the duodenal concentrations of CEX at 5 and 10 min were slightly lower than those of FD-4 due to CEX absorption, the duodenal CEX concentrations exceeded FD-4 concentrations at timescales longer than $20 \mathrm{~min}$ after oral administration (Figure 1). This may be due to biliary secretion of CEX into the duodenum (27). However, CEX did not exceed the FD-4 concentration in the upper jejunum, indicating that secreted CEX was rapidly re-absorbed. Ultimately, CEX was completely absorbed from the GI tract. Since PEPT1 is expressed throughout the rat small intestine (28), it is reasonable that $\mathrm{CEX}$ was efficiently absorbed from the small intestine. This observation is consistent with the results of the BA-time profile that showed continuous absorption and high BA of CEX $(85.2 \%)$ (Figure 5).

The fraction absorbed of SQV until it reached the upper jejunum and ileum was calculated based on the area under the luminal concentration-time curve of FD-4 (AUC $\left.\mathrm{AI}_{\mathrm{FD}-4)}\right)$ and $\mathrm{SQV}\left(\mathrm{AUC}_{\mathrm{GI}(\mathrm{SQV})}\right)$ until 150 min according to our previous study (11). Since the difference between $\mathrm{AUC}_{\mathrm{GI}(\mathrm{FD}-4)}$ and $\mathrm{AUC}_{\mathrm{GI}(\mathrm{SQV})}$ reflects to absorbed amount of SQV in these intestinal segments, the fraction absorbed of SQV (\%) can be calculated as follows: Fraction absorbed

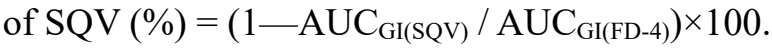

The estimation of the fraction absorbed revealed that $42.8 \%$ of administered SQV was absorbed until it reached upper jejunum, but only $20.1 \%$ of SQV was absorbed from the upper jejunum to ileum (The overall fraction absorbed of SQV was $62.9 \%$ ). This is probably because that the absorption of SQV was impeded due to the efflux via P-gp in the distal region. The luminal concentration data was supported by the result of BA-time profile of SQV that showed rapid absorption of SQV at early time period (Figure 5). It has been reported that SQV is also a substrate for MRP2 that is expressed in the upper small intestine (2). However, since SQV was primarily absorbed from the upper region, contribution of MRP2 for SQV efflux may be low compared to P-gp.

The BA-time profile of MTX indicated that MTX was primarily absorbed in the upper region of the small intestine (Figure 5). Yokooji et al. have reported that MTX absorption in the proximal small intestine was higher than that found in the distal small intestine in rats (12). Their findings are consistent with our results. They also revealed that the site-specific absorption of MTX was primarily caused by the local expression of PCFT, an absorptive transporter for MTX, in the upper region of the small intestine (12), although other transporters (e.g., RFC1, MRP2, P-gp and BCRP) are also involved in MTX absorption. To clarify the actual contribution of each transporter to the absorption, further experiments using a specific transporter inhibitor or transporter-knockout animals are required.

The BA-time profile of MTX corresponded to the result of luminal concentration patterns of MTX (Figure 3). In the duodenum, the concentration of MTX exceeded the FD-4 concentration over the timeframe of 10-150 min (Figure 3). This was due to biliary secretion of MTX into the duodenal segment $(29,30)$. 

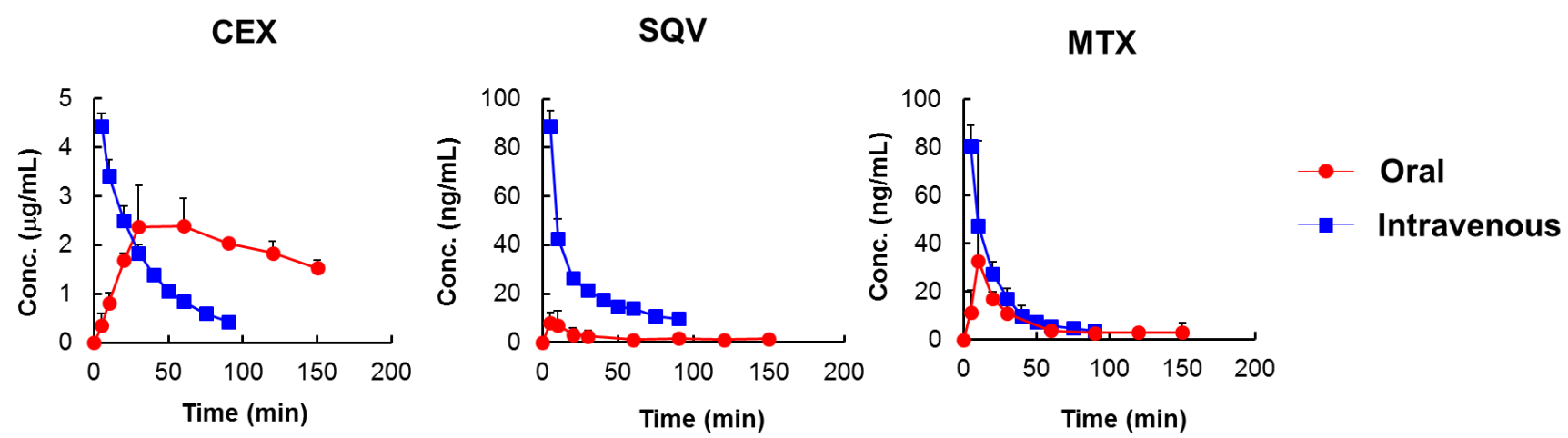

Figure 4. Time-courses of plasma concentration after oral and intravenous administration of various drugs. The doses of CEX, SQV, and MTX were $1.0,0.2$, and $0.025 \mathrm{mg} /$ rat for oral administration and $0.5,0.1$, and $0.0125 \mathrm{mg} / \mathrm{rat}$ for intravenous administration, respectively. The data are expressed as the mean of each experiment with S.D. (n=3-4).
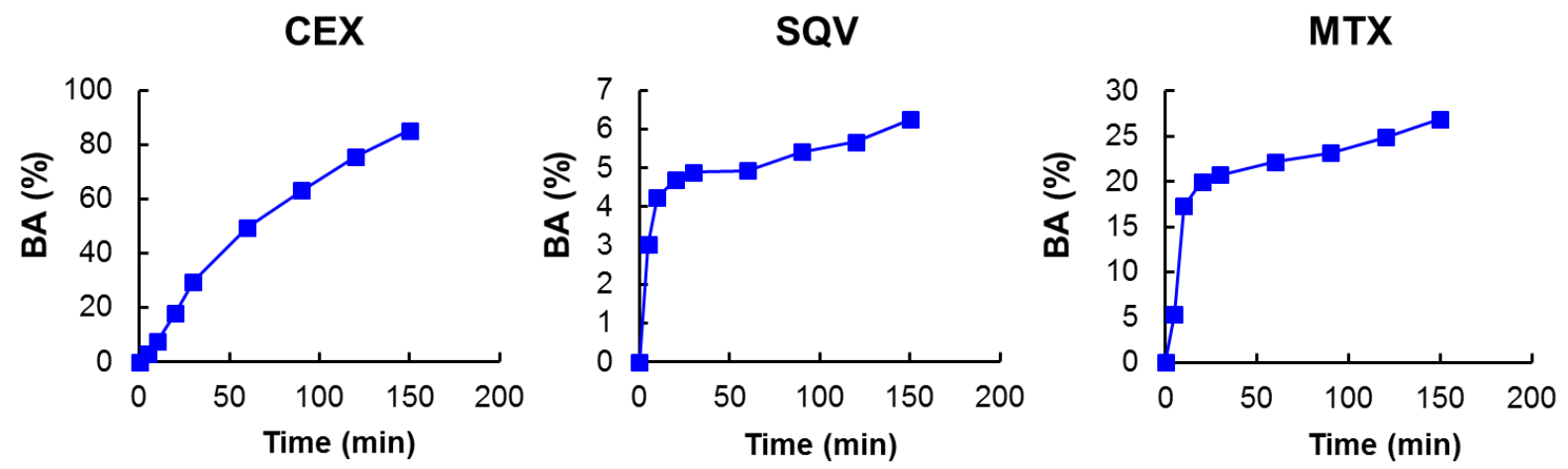

Figure 5. Time-course of BA after oral adminstration of various drugs, as calculated by the deconvolution method.

However, the MTX concentration lowered to the same extent as FD-4 concentration after $10 \mathrm{~min}$ in the upper jejunum. This indicated that the MTX was absorbed via PCFT and RFC1 (RFC1 is expressed in whole small intestine (19)) from the duodenum to upper jejunum. However, the difference in the concentration between FD-4 and MTX that reflects to fraction absorbed was almost unchanged from the upper jejunum to lower jejunum (i.e., MTX was almost not absorbed in these intestinal regions), probably due to efflux via P-gp and BCRP (BCRP is expressed in the whole small intestine (2)). Also, the concentration in the lower jejunum and ileum exceeded the dosing concentration, similar to FD-4, due to condensation by fluid absorption.

The guidelines for in vitro drug interaction studies from the FDA state that the investigated drug should be investigated to determine whether it is a substrate of $\mathrm{P}$-gp and BCRP using in vitro transport experiments (31). For P-gp, since the main expression site is the lower region of the small intestine, the drug concentration pattern in this region should be considered for accurate evaluation and prediction of P-gp-mediated drug interactions. The same is true for other transporters with specific expressions (e.g., PCFT). In addition, in the case of low-permeability drugs, attention should be paid to the condensation of drugs induced by fluid absorption.

\section{CONCLUSIONS}

The intraluminal behavior of drugs is often a black box. This study revealed the luminal concentration patterns of various transporter substrates after oral administration. We further examined the absorption behavior based on differences in the FD-4 and drug concentrations. The concentration-time profiles differed depending on the differences in the absorption properties at different sites for each drug and fluid volume in the individual GI segments. Since drug absorption pattern is determined by the 
luminal drug concentration pattern, our findings are useful for setting the initial drug concentration for in vitro permeation studies to determine the actual effect of transporters on absorption and in silico predictions.

CONFLICT OF INTEREST. We have no financial or non-financial interests that represent conflicts of interest.

FUNDING. This research received no specific grant from any funding agency in the public, commercial, or not-for-profit sectors.

\section{REFERENCES}

1. Tamai I. Oral drug delivery utilizing intestinal OATP transporters. Adv Drug Deliv Rev, 2012; 64:508-14.

2. Takano M, Yumoto R, Murakami T. Expression and function of efflux drug transporters in the intestine. Pharmacol Ther, 2006; 109:137-161.

3. Varma MV, Ambler CM, Ullah M, Rotter CJ, Sun H, Litchfield J, Fenner KS, El-Kattan AF. Targeting intestinal transporters for optimizing oral drug absorption. Curr Drug Metab, 2010; 11:730-42.

4. Kwon H, Lionberger RA, Yu LX. Impact of Pglycoprotein-mediated intestinal efflux kinetics on oral bioavailability of Pglycoprotein substrates. Mol Pharm, 2004; 1:455-65.

5. Shirasaka Y, Sakane T, Yamashita S. Effect of P-glycoprotein expression levels on the concentration-dependent permeability of drugs to the cell membrane. J Pharm Sci, 2008; 97:553-65.

6. Ayrton A, Morgan P. Role of transport proteins in drug absorption, distribution and excretion. Xenobiotica, 2001; 31:469-97.

7. Wetterich U, Spahn-Langguth H, Mutschler E, Terhaag B, Rösch W, Langguth P. Evidence for intestinal secretion as an additional clearance pathway of talinolol enantiomers: concentration- and dose-dependent absorption in vitro and in vivo. Pharm Res, 1996; 13:51422.

8. Tomaru A, Takeda-Morishita M, Maeda K, Banba H, Takayama K, Kumagai Y, Kusuhara H, Sugiyama Y. Effects of Cremophor EL on the absorption of orally administered saquinavir and fexofenadine in healthy subjects. Drug Metab Pharmacokinet, 2015; 30:221-226.

9. Döppenschmitt S, Spahn-Langguth $H$, Regårdh CG, Langguth $\mathrm{P}$. Role of Pglycoprotein-mediated secretion in absorptive drug permeability: An approach using passive membrane permeability and affinity to Pglycoprotein. J Pharm Sci, 1999; 88:1067-72.

10. Tanaka Y, Higashino H, Kataoka M, Yamashita S. In vivo fluid volume in rat gastrointestinal tract: kinetic analysis on the luminal concentration of non-absorbable FITC-dextran after oral administration. J Pharm Sci, 2020; 109:1863-1866.

11. Tanaka Y, Goto T, Kataoka M, Sakuma S, Yamashita S. Impact of Luminal Fluid Volume on the Drug Absorption After Oral Administration: Analysis Based on In Vivo Drug Concentration-Time Profile in the Gastrointestinal Tract. J Pharm Sci, 2015; 104:3120-3127.

12. Yokooji T, Mori N, Murakami T. Site-specific contribution of proton-coupled folate transporter/haem carrier protein 1 in the intestinal absorption of methotrexate in rats. $\mathrm{J}$ Pharm Pharmacol, 2009; 61:911-8.

13. Brouwers J, Augustijns P. Resolving intraluminal drug and formulation behavior: Gastrointestinal concentration profiling in humans. Eur J Pharm Sci. 2014; 61:2-10.

14. Yamashita S, Kataoka M, Higashino $H$, Sakuma S, Sakamoto T, Uchimaru H, Tsukikawa H, Shiramoto M, Uchiyama H, Tachiki H, Irie S. Measurement of drug concentration in the stomach after intragastric administration of drug solution to healthy volunteers: analysis of intragastric fluid dynamics and drug absorption. Pharm Res. 2013; 30:951-8.

15. Kourentas A, Vertzoni M, Symillides $M$, Goumas K, Gibbon R, Butler J, Reppas C. Effectiveness of supersaturation promoting excipients on albendazole concentrations in upper gastrointestinal lumen of fasted healthy adults. Eur J Pharm Sci. 2016; 91:11-9.

16. Tanaka Y, Tay E, Nguyen TH, Porter CJH. Quantifying In Vivo Luminal Drug Solubilization-Supersaturation-Precipitation Profiles to Explain the Performance of Lipid Based Formulations. Pharm. Res. 2020; 37:47.

17. Hironaka T, Itokawa S, Ogawara K, Higaki K, Kimura T. Quantitative evaluation of PEPT1 contribution to oral absorption of cephalexin 
in rats. Pharm Res, 2009; 26:40-50.

18. Buchanan CM, Buchanan NL, Edgar KJ, Little JL, Ramsey MG, Ruble KM, Wacher VJ, Wempe MF. Pharmacokinetics of saquinavir after intravenous and oral dosing of saquinavir: hydroxybutenyl-beta-cyclodextrin formulations. Biomacromolecules, 2008; 9:305-13.

19. Chiao JH, Roy K, Tolner B, Yang CH, Sirotnak FM. RFC-1 gene expression regulates folate absorption in mouse small intestine. $\mathrm{J}$ Biol Chem, 1997; 272:11165-70.

20. Breedveld P, Zelcer N, Pluim D, Sönmezer O, Tibben MM, Beijnen JH, Schinkel AH, van Tellingen $\mathrm{O}$, Borst $\mathrm{P}$, Schellens JH. Mechanism of the pharmacokinetic interaction between methotrexate and benzimidazoles: potential role for breast cancer resistance protein in clinical drug-drug interactions. Cancer Res, 2004; 64:5804-11.

21. Wang L, Wang C, Peng J, Liu Q, Meng Q, Sun H, Huo X, Sun P, Yang X, Zhen Y, Liu K. Dioscin enhances methotrexate absorption by down-regulating MDR1 in vitro and in vivo. Toxicol Appl Pharmacol, 2014; 277:146-54.

22. Zhu Y, Meng Q, Wang C, Liu Q, Huo X, Zhang A, Sun P, Sun H, Li H, Liu K. Methotrexatebestatin interaction: involvement of $\mathrm{P}$ glycoprotein and organic anion transporters in rats. Int J Pharm, 2014; 465:368-77.

23. Dressman, J. B.; Lennernäs H., Oral Drug Absorption : Prediction and Assessment. Marcel Dekker, New York, USA, 2000.

24. Tanaka Y, Hara T, Waki R, Nagata S. Regional differences in the components of luminal water from rat gastrointestinal tract and comparison with other species. J Pharm Pharm Sci, 2012; 15:510-8.

25. Lindahl A, Ungell AL, Knutson L, Lennernäs $\mathrm{H}$. Characterization of fluids from the stomach and proximal jejunum in men and women. Pharm Res, 1997; 14:497-502.

26. Tanaka Y, Nguyen TH, Suys EJA, Porter CJH. Digestion of Lipid-Based Formulations Not Only Mediates Changes to Absorption of Poorly Soluble Drugs Due to Differences in Solubilization But Also Reflects Changes to Thermodynamic Activity and Permeability. Mol. Pharm, 2021; 18:1768-1778.

27. Barrio Lera JP, Alvarez AI, Prieto JG. Effects of ethanol on the pharmacokinetics of cephalexin and cefadroxil in the rat. J Pharm Sci, 1991; 80: 511-6.
28. Tomohiro T. Kyoto University Research Information Repository, 1999; Available at: https://repository.kulib.kyotou.ac.jp/dspace/bitstream/2433/73163/1/D Ter ada Tomohiro.pdf.

29. Kuroda T, Namba K, Torimaru T, Kawashima $\mathrm{K}$, Hayashi M. Species differences in oral bioavailability of methotrexate between rats and monkeys. Biol Pharm Bull, 2000; 23:3348.

30. Bremnes RM, Slørdal L, Wist E, Aarbakke J. Dose-dependent pharmacokinetics of methotrexate and 7-hydroxymethotrexate in the rat in vivo. Cancer Res, 1989; 49:6359-64.

31. U.S. Department of Health and Human Services Food and Drug Administration Center for Drug Evaluation and Research. 2020. Guidance for Industry: In Vitro Drug Interaction Studies - Cytochrome P450 Enzyme- and Transporter-Mediated Drug Interactions. Available at: https://www.fda.gov/media/134582/download. 